\title{
Pricing and Remanufacturing Decisions of a Decentralized Fuzzy Supply Chain
}

\author{
Jing Zhao, ${ }^{1}$ Weiyu Liu, ${ }^{1}$ and Jie Wei ${ }^{2}$ \\ ${ }^{1}$ School of Science, Tianjin Polytechnic University, Tianjin 300160, China \\ ${ }^{2}$ General Courses Department, Military Transportation University, Tianjin 300161, China \\ Correspondence should be addressed to Jie Wei; weijie2288@163.com
}

Received 7 November 2012; Accepted 31 December 2012

Academic Editor: Xiaochen Sun

Copyright (c) 2013 Jing Zhao et al. This is an open access article distributed under the Creative Commons Attribution License, which permits unrestricted use, distribution, and reproduction in any medium, provided the original work is properly cited.

\begin{abstract}
The optimal pricing and remanufacturing decisions problem of a fuzzy closed-loop supply chain is considered in this paper. Particularly, there is one manufacturer who has incorporated a remanufacturing process for used products into her original production system, so that she can manufacture a new product directly from raw materials or from collected used products. The manufacturer then sells the new product to two different competitive retailers, respectively, and the two competitive retailers are in charge of deciding the rates of the remanufactured products in their consumers' demand quantity. The fuzziness is associated with the customer's demands, the remanufacturing and manufacturing costs, and the collecting scaling parameters of the two retailers. The purpose of this paper is to explore how the manufacturer and the two retailers make their own decisions about wholesale price, retail prices, and the remanufacturing rates in the expected value model. Using game theory and fuzzy theory, we examine each firm's strategy and explore the role of the manufacturer and the two retailers over three different game scenarios. We get some insights into the economic behavior of firms, which can serve as the basis for empirical study in the future.
\end{abstract}

\section{Introduction}

In recent years, the management of closed-loop supply chains has gained growing attention from both business and academic research because of environmental consciousness, environmental concerns, and stringent environmental laws, for example, the legislation on producer responsibility, requiring companies to take back products from customers and to organize for proper recovery and disposal. This legislation is partially due to increased awareness of environmental issues. However, smart companies have also understood that used products often contain lots of value to be recovered. They manage closed-loop supply chains simply because it is a profitable business proposition. It is said that the costs derived from reverse-logistics activities in the USA exceed $\$ 35$ billion per year; remanufacturing is a $\$ 53$ billion industry in the USA [1].

Without a doubt, closed-loop supply chains has become a matter of strategic importance: an element that companies must consider in decision-making processes concerning the design and development of their supply chains [2]. A specific type of closed-loop supply chains is product manufacturing and remanufacturing supply chain. Product remanufacturing is the process that restores used products or product parts to an "as good as new" condition, after which they can be resold on the market of new products. The industrial operations involved with remanufacturing are of a very uncertain nature due to the uncertainty in timing, quantity, and quality of collected products. So one of the important management issues in product manufacturing and remanufacturing closed-loop supply chains is to effectively match demand, and supply by dealing with the uncertainty of the quality and quantities of the collected products and of the market demand.

In fact, in order to make effective closed-loop supply chain management, the uncertainties that happen in the real world cannot be ignored. Those uncertainties are usually associated with the product supply, used product collecting, the customer demand, and so on. Traditional probabilistic concepts have been used to model the various parameters among today's many studies published on the reverse logistics [3-5]. However, the probability-based approaches may not be sufficient enough to reflect all uncertainties that may arise 
in a real world manufacturing and remanufacturing closedloop supply chains. Modelers may face some difficulties while trying to build a valid model of a manufacturing and remanufacturing closed-loop supply chains, in which the related costs cannot be determined precisely. For example, costs may be dependent on some foreign monetary unit, current interest rate, stock keeping unit's market price, and the quality of collected product, which may not be known precisely. Since some uncertainty within manufacturing and remanufacturing closed-loop supply chains cannot be considered appropriately using concepts of probability theory, the quantitative demand forecasts based on manager's judgements, intuitions, and experience seem to be more appropriate, and fuzzy theory rather than probability theory should be applied to model this kind of uncertainties [6]. Fuzzy theory provides a reasonable way to deal with the possibility and linguistic expressions. Zadeh [7] initialized the concept of a fuzzy set via membership function. From then on, many researchers such as Nahmias [8] and Kaufmann and Gupta [9] made great contributions to this field. Recently, Liu [10] B. Liu and Y. K. Liu [11] laid a new foundation for optimization problems in the fuzzy environment, in which the expected value was proposed to deal with optimization problems.

In recent supply chain studies, some researchers have already adopted fuzzy theory to depict uncertainties in supply chain models [12-16]. Li et al. [17] obtained the optimal order quantity for the fuzzy newsboy models through fuzzy ordering of fuzzy numbers with respect to their total integral values. Mukhopadhyay and Ma [18] addressed the issue of a hybrid system where both used and new parts can serve as inputs in the production process to satisfy an uncertain market demand. Kao and Hsu [19] proposed a newsboy model for cases of fuzzy demand. They obtained the optimal policy to minimize the total cost by adopting a method for ranking fuzzy numbers.

Although some researches on the forward supply chain have been given through considering the supply chain's fuzzy uncertainties, little researches on the reverse supply chain considering the fuzzy uncertainties has been established to our knowledge. So, in this paper, we consider a fuzzy manufacturing and remanufacturing closed-loop supply chain with one manufacturer and two competitive retailers; the fuzziness is associated with the consumer demand, the manufacturing and remanufacturing costs of new product, and the collecting cost of the used product. In the forward supply chain, the manufacturer has incorporated a remanufacturing process for used products into her original production system, so that she can manufacture a new product directly from raw materials, or remanufacture part or whole of a collected unit, and wholesales the new products to the two competitive retailers who then sell them to the end consumers. For the the reverse supply chain, the two competitive retailers are in charge of collecting the used products from the consumers, respectively. Using game theory and fuzzy theory, the optimal decisions for each supply chain participant are explored in the expected value model. Some management insights are given in this paper.

The rest of the paper is organized as follows. Section 2 gives the problem description and notations, and Section 3 details our key analytical results. Numerical studies are given in Section 4. Concluding remarks are presented in Section 5.

\section{Problem Description}

Consider a closed-loop supply chain in a fuzzy environment with one manufacturer and two competitive retailers, labeled retailer 1 and retailer 2 . In the following discussion, "he" represents one of the two manufacturers, and "she" represents the retailer. In the forward supply chain, similar to Savaskan et al. [20], assume that the manufacturer has incorporated a remanufacturing process for used products into her original production system, so he can manufacture a new product directly from raw materials with unit manufacturing $\operatorname{cost} \widetilde{c}_{m}$, or from collected products with unit remanufacturing cost $\widetilde{c}_{r} . \widetilde{c}_{m}$ and $\widetilde{c}_{r}$ are all fuzzy variables. (For the preliminaries of fuzzy theory used in this paper see the preliminaries in [16]). The manufacturer wholesales the new product to the two competitive retailers, respectively, with unit wholesale price $w$, then the two competitive retailers sell them to the consumers with unit retail price $p_{i}$, which is a decision variable of retailer $i$. We assume that the two retailers are equally powerful and compete in one common market, and all activities occur within a single period. The two competitive retailers face fuzzy linear consumer demands that are influenced by the retail prices of the new product made by the two retailers, respectively. The manufacturer and the two competitive retailers must make their pricing strategies in order to achieve optimal expected profits and behave as if they have perfect information of the demands and the cost structures of other channel members. In the reverse supply chain, the two competitive retailers are in charge of deciding the collecting rates of the remanufactured products in the consumers' demand quantity, denoted as $\tau_{i}$, and taking back the used products from the end consumers with taking back cost $c\left(\tau_{i}\right)(i=1,2)$, according to our survey results; assume that $c\left(\tau_{i}\right)=\widetilde{k}_{i} \tau_{i}^{2}$, where $\widetilde{k}_{i}$ is a scaling parameter, which is a fuzzy variable. The manufacturer will take back all the used products collected by the two competitive retailers with unit transfer $\operatorname{cost} \widetilde{c}_{f}$, which is a fuzzy variable.

We define the retailer $i$ 's price-dependent demand a

$$
D_{i}\left(p_{i}, p_{j}\right)=\widetilde{a}-p_{i}+\widetilde{\beta} p_{j}, \quad i=1,2, \quad j=3-i,
$$

where $\widetilde{a}, \widetilde{\beta}$ are nonnegative fuzzy variables, $\widetilde{a}$ denotes the primary demand of retailer $i$ 's product, $\widetilde{\beta}$ denotes the measure of the responsiveness of each retailer's product's market demand to its competitor's price. We assume that the fuzzy linear demand (1) is symmetrical. This represents a situation in which two retailers have equal competing power in a duopolistic marketplace. We assume that $E[\widetilde{\beta}]<1$, which ensures that the response functions are negatively sloped which, in turn, ensures the existence of the equilibrium solutions. This seems reasonable since sales are relatively more sensitive to price at a retailer's own outlet(s) than at the competing retailer's outlets. In the past, similar demand function has been used widely in marketing research literature (see [21-24]) and in some economic literature (see [2527]). Moreover, in this paper, assume that fuzzy variables $\widetilde{c}_{m}$, 
$\widetilde{c}_{r}, \widetilde{a}, \widetilde{\beta}, \widetilde{c}_{f}, \widetilde{k}_{1}, \widetilde{k}_{2}$ are all independently nonnegative, which is reasonable in the real world.

In our models, the manufacturer can influence the demand by setting the new product's wholesale price; the two competitive retailers can independently decide the retail price of the new product and the collecting rate of the used product. We do not assume any collusion or cooperation among firms; this assumption is typical in analytical model, although it overstates the information climate of the real world. The logistic cost components of the manufacturer and two retailers (e.g., carrying cost inventory cost, etc.) are without consideration for analytical convenience.

Assume each channel member has the same goal: to maximize his/her own expected profit. From the above descriptions, the two competitive retailers' objectives are to maximize their own expected profits (denoted as $E\left[\pi_{r_{i}}\right]$ ), which can be described as follows:

$$
\begin{aligned}
\operatorname{Max}_{p_{i}, \tau_{i}} E\left[\pi_{r_{i}}\right]=\operatorname{Max}_{p_{i}, \tau_{i}} E[ & \left(p_{i}-w\right) D_{i}\left(p_{i}, p_{j}\right) \\
& \left.-\tilde{k}_{i} \tau_{i}^{2}+\widetilde{c}_{f} \tau_{i} D_{i}\left(p_{i}, p_{j}\right)\right],
\end{aligned}
$$

where

$$
\pi_{r_{i}}=\left(p_{i}-w\right) D_{i}\left(p_{i}, p_{j}\right)-\widetilde{k}_{i} \tau_{i}^{2}+\widetilde{c}_{f} \tau_{i} D_{i}\left(p_{i}, p_{j}\right) .
$$

The manufacturer's objective is to maximize his own expected profit (denoted as $E\left[\pi_{m}\right]$ ), which can be described as follows:

$$
\begin{aligned}
\operatorname{Max}_{w} E\left[\pi_{m}\right] & \\
=\operatorname{Max}_{w} E[ & \left(w-\left(\widetilde{c}_{f}-\widetilde{c}_{m}+\widetilde{c}_{r}\right) \tau_{1}-\widetilde{c}_{m}\right) D_{1}\left(p_{1}, p_{2}\right) \\
& \left.+\left(w-\left(\widetilde{c}_{f}-\widetilde{c}_{m}+\widetilde{c}_{r}\right) \tau_{2}-\widetilde{c}_{m}\right) D_{2}\left(p_{2}, p_{1}\right)\right],
\end{aligned}
$$

where

$$
\begin{aligned}
\pi_{m}= & \left(w-\left(\widetilde{c}_{f}-\widetilde{c}_{m}+\widetilde{c}_{r}\right) \tau_{1}-\widetilde{c}_{m}\right) D_{1}\left(p_{1}, p_{2}\right) \\
& +\left(w-\left(\widetilde{c}_{f}-\widetilde{c}_{m}+\widetilde{c}_{r}\right) \tau_{2}-\widetilde{c}_{m}\right) D_{2}\left(p_{2}, p_{1}\right) .
\end{aligned}
$$

Note that so far we have not made any assumptions regarding the bargaining power possessed by each channel member. The assumption regarding bargaining power possessed by each firm can influence how the pricing game is solved in our model. Variation in bargaining power in a particular supply chain can create one of the following three scenarios: (1) Manufacturer Stackelberg: the manufacturer has more bargaining power than the two competitive retailers and thus is the Stackelberg leader. (2) Retailer Stackelberg: the two competitive retailers have more bargaining power than the manufacturer and are the Stackelberg leaders. (3) Vertical Nash: every firm in the system has equal bargaining power.

\section{Model Analysis}

To analyze our model, we follow a game theory approach. The leader in each scenario makes his decision to maximize his/her own expected profit, conditioned on the follower's response. The problem can be solved backwards. We begin by first solving for the decision of the follower of the game, given that he/she has observed the leader's decision. For example, in Manufacturer Stackelberg, the two competitive retailers' decisions are derived first, given that the two competitive retailers have observed the decision made by the manufacturer (on wholesale price). Then, the manufacturer solves his problem given that he knows how the two competitive retailers would react to his decision.

\subsection{Manufacturer Stackelberg}

3.1.1. Retailers' Decisions. In the Manufacturer Stackelberg game case, the manufacturer first announces his wholesale prices of the new product. The two competitive retailers observe the wholesale price and then simultaneously decide the retail prices they are going to charge for their own product and the collecting rates of the used products. Note that the two competitive retailers move simultaneously. Therefore, we need to calculate the Nash decisions between them first.

Proposition 1. The two competitive retailers' optimal retail prices and optimal collecting rates of used products, given earlier decision $w$ made by the manufacturer, are

$$
\begin{aligned}
p_{1}^{*} & =\frac{B_{1}}{A} w+\frac{B_{2}}{A}, \\
p_{2}^{*} & =\frac{B_{3}}{A} w+\frac{B_{4}}{A}, \\
\tau_{1}^{*} & =E_{1} w+E_{2}, \\
\tau_{2}^{*} & =E_{3} w+E_{4},
\end{aligned}
$$

where

$$
\begin{aligned}
A= & \left(2 E\left[\widetilde{k}_{2}\right] E[\tilde{\beta}]-E\left[\widetilde{c}_{f} \tilde{\beta}\right] E\left[\widetilde{c}_{f}\right]\right) \\
& \times\left(2 E\left[\widetilde{k}_{1}\right] E[\widetilde{\beta}]-E\left[\widetilde{c}_{f} \tilde{\beta}\right] E\left[\widetilde{c}_{f}\right]\right) \\
& -\left(E^{2}\left[\widetilde{c}_{f}\right]-4 E\left[\widetilde{k}_{1}\right]\right)\left(E^{2}\left[\widetilde{c}_{f}\right]-4 E\left[\widetilde{k}_{2}\right]\right), \\
B_{1}= & 2 E\left[\widetilde{k}_{1}\right]\left(E^{2}\left[\widetilde{c}_{f}\right]-4 E\left[\widetilde{k}_{2}\right]\right) \\
& -2 E\left[\widetilde{k}_{2}\right]\left(2 E\left[\widetilde{k}_{1}\right] E[\widetilde{\beta}]-E\left[\widetilde{c}_{f} \widetilde{\beta}\right] E\left[\widetilde{c}_{f}\right]\right), \\
B_{2}= & \left(2 E\left[\widetilde{k}_{1}\right] E[\widetilde{a}]-E\left[\widetilde{c}_{f} \widetilde{a}\right] E\left[\widetilde{c}_{f}\right]\right) \\
& \times\left(E^{2}\left[\widetilde{c}_{f}\right]-4 E\left[\widetilde{k}_{2}\right]\right) \\
& -\left(2 E\left[\widetilde{k}_{2}\right] E[\widetilde{a}]-E\left[\widetilde{c}_{f} \widetilde{a}\right] E\left[\widetilde{c}_{f}\right]\right) \\
& \times\left(2 E\left[\widetilde{k}_{1}\right] E[\widetilde{\beta}]-E\left[\widetilde{c}_{f} \widetilde{\beta}\right] E\left[\widetilde{c}_{f}\right]\right),
\end{aligned}
$$




$$
\begin{aligned}
& B_{3}=2 E\left[\widetilde{k}_{2}\right]\left(E^{2}\left[\widetilde{\boldsymbol{c}}_{f}\right]-4 E\left[\widetilde{k}_{1}\right]\right) \\
& -2 E\left[\widetilde{k}_{1}\right]\left(2 E\left[\widetilde{k}_{2}\right] E[\widetilde{\beta}]-E\left[\widetilde{c}_{f} \widetilde{\beta}\right] E\left[\widetilde{c}_{f}\right]\right) \text {, } \\
& B_{4}=\left(2 E\left[\widetilde{k}_{2}\right] E[\tilde{a}]-E\left[\widetilde{c}_{f} \tilde{a}\right] E\left[\widetilde{c}_{f}\right]\right) \\
& \times\left(E^{2}\left[\widetilde{c}_{f}\right]-4 E\left[\widetilde{k}_{1}\right]\right) \\
& -\left(2 E\left[\widetilde{k}_{1}\right] E[\tilde{a}]-E\left[\widetilde{c}_{f} \tilde{a}\right] E\left[\widetilde{c}_{f}\right]\right) \\
& \times\left(2 E\left[\tilde{k}_{2}\right] E[\tilde{\beta}]-E\left[\widetilde{c}_{f} \tilde{\beta}\right] E\left[\widetilde{c}_{f}\right]\right), \\
& E_{1}=\frac{1}{E\left[\widetilde{c}_{f}\right]}\left(1-\frac{2 B_{1}}{A}+\frac{E[\tilde{\beta}] B_{3}}{A}\right) \text {, } \\
& E_{2}=\frac{1}{E\left[\tilde{c}_{f}\right]}\left(E[\tilde{a}]-\frac{2 B_{2}}{A}+\frac{E[\widetilde{\beta}] B_{4}}{A}\right) \text {, } \\
& E_{3}=\frac{1}{E\left[\widetilde{c}_{f}\right]}\left(1-\frac{2 B_{3}}{A}+\frac{E[\widetilde{\beta}] B_{1}}{A}\right) \text {, } \\
& E_{4}=\frac{1}{E\left[\tilde{c}_{f}\right]}\left(E[\tilde{a}]-\frac{2 B_{4}}{A}+\frac{E[\tilde{\beta}] B_{2}}{A}\right) .
\end{aligned}
$$

Proof. Using (3), we can have the expected value of $\pi_{r_{i}}$ as follows:

$$
\begin{aligned}
E\left[\pi_{r_{i}}\right]= & \left(p_{i}-w\right)\left(E[\widetilde{a}]-p_{i}+E[\widetilde{\beta}] p_{j}\right)-E\left[\widetilde{k}_{i}\right] \tau_{i}^{2} \\
& +E\left[\widetilde{c}_{f} \widetilde{a}\right] \tau_{i}-E\left[\widetilde{c}_{f}\right] \tau_{i} p_{i}+E\left[\widetilde{c}_{f} \widetilde{\beta}\right] \tau_{i} p_{j} .
\end{aligned}
$$

From (11), the first order partial derivatives of $E\left[\pi_{r_{1}}\right]$ to $p_{1}, \tau_{1}$ and $E\left[\pi_{r_{2}}\right]$ to $p_{2}, \tau_{2}$ can be shown as

$$
\begin{aligned}
& \frac{\partial E\left[\pi_{r_{1}}\right]}{\partial p_{1}}=w-2 p_{1}+E[\widetilde{a}]+E[\tilde{\beta}] p_{2}-E\left[\widetilde{c}_{f}\right] \tau_{1}, \\
& \frac{\partial E\left[\pi_{r_{1}}\right]}{\partial \tau_{1}}=-2 E\left[\widetilde{k}_{1}\right] \tau_{1}+E\left[\widetilde{c}_{f} \widetilde{a}\right]-E\left[\widetilde{c}_{f}\right] p_{1}+E\left[\widetilde{c}_{f} \widetilde{\beta}\right] p_{2}, \\
& \frac{\partial E\left[\pi_{r_{2}}\right]}{\partial p_{2}}=w-2 p_{2}+E[\widetilde{a}]+E[\tilde{\beta}] p_{1}-E\left[\widetilde{c}_{f}\right] \tau_{2}, \\
& \frac{\partial E\left[\pi_{r_{2}}\right]}{\partial \tau_{2}}=-2 E\left[\tilde{k}_{2}\right] \tau_{2}+E\left[\widetilde{c}_{f} \tilde{a}\right]-E\left[\widetilde{c}_{f}\right] p_{2}+E\left[\widetilde{c}_{f} \tilde{\beta}\right] p_{1} .
\end{aligned}
$$

Then, we can have the first order conditions as follows:

$$
\begin{gathered}
w-2 p_{1}+E[\widetilde{a}]+E[\widetilde{\beta}] p_{2}-E\left[\widetilde{c}_{f}\right] \tau_{1}=0, \\
-2 E\left[\widetilde{k}_{1}\right] \tau_{1}+E\left[\widetilde{c}_{f} \widetilde{a}\right]-E\left[\widetilde{c}_{f}\right] p_{1}+E\left[\widetilde{c}_{f} \widetilde{\beta}\right] p_{2}=0, \\
w-2 p_{2}+E[\widetilde{a}]+E[\widetilde{\beta}] p_{1}-E\left[\widetilde{c}_{f}\right] \tau_{2}=0, \\
-2 E\left[\widetilde{k}_{2}\right] \tau_{2}+E\left[\widetilde{c}_{f} \widetilde{a}\right]-E\left[\widetilde{c}_{f}\right] p_{2}+E\left[\widetilde{c}_{f} \tilde{\beta}\right] p_{1}=0 .
\end{gathered}
$$

Solving (13), simultaneously, we can easily have (6)-(9), so Proposition 1 is proven.

3.1.2. Manufacturer's Decision. The manufacturer in this game is the Stackelberg leader. He announces his new product's wholesale price $w$. Using the retailers' decisions, we can derive the manufacturer's optimal wholesale price. This is carried out by maximizing the manufacturer's expected profit $E\left[\pi_{m}\right]$, given the two competitive retailers' decisions, which are given as in Proposition 1. The manufacturer chooses the wholesale price $w$ to maximize his own individual expected profit $E\left[\pi_{m}\right]$, which can be given as follows:

$$
\begin{aligned}
\operatorname{Max}_{w} E\left[\pi_{m}\right] & \\
=\operatorname{Max}_{w} E[ & \left(w-\left(\widetilde{c}_{f}-\widetilde{c}_{m}+\widetilde{c}_{r}\right) \tau_{1}^{*}-\widetilde{c}_{m}\right) D_{1}\left(p_{1}^{*}, p_{2}^{*}\right) \\
& \left.+\left(w-\left(\widetilde{c}_{f}-\widetilde{c}_{m}+\widetilde{c}_{r}\right) \tau_{2}^{*}-\widetilde{c}_{m}\right) D_{2}\left(p_{2}^{*}, p_{1}^{*}\right)\right],
\end{aligned}
$$

where $p_{1}^{*}, p_{2}^{*}, \tau_{1}^{*}, \tau_{2}^{*}$ are defined as in (6)-(9), respectively.

Proposition 2. In the Manufacturer Stackelberg game case, the manufacturer's optimal decision (denoted as $w_{m}^{*}$ ) is satisfied as follows:

$$
\begin{aligned}
& 2 E[\widetilde{a}]+\frac{\left(B_{2}+B_{4}\right)(E[\widetilde{\beta}]-1)}{A}+2(E[\widetilde{\beta}]-1) \frac{B_{1}+B_{3}}{A} w_{m}^{*} \\
& -\left(\frac{1}{2} \int_{0}^{1}\left(\widetilde{a}_{\alpha}^{U} \widetilde{c}_{f \alpha}^{L}+\widetilde{a}_{\alpha}^{L} \widetilde{c}_{f \alpha}^{U}\right) d \alpha-E\left[\widetilde{a}_{m}\right]\right. \\
& \left.+\frac{1}{2} \int_{0}^{1}\left(\widetilde{a}_{\alpha}^{U} \widetilde{c}_{r \alpha}^{L}+\widetilde{a}_{\alpha}^{L} \widetilde{c}_{r \alpha}^{U}\right) d \alpha\right) E_{1} \\
& +E\left[\widetilde{c}_{m}\right] \frac{B_{1}}{A}-\frac{B_{3}}{2 A} \int_{0}^{1}\left(\widetilde{\beta}_{\alpha}^{U} \widetilde{c}_{m \alpha}^{L}+\widetilde{\beta}_{\alpha}^{L} \widetilde{c}_{m \alpha}^{U}\right) d \alpha \\
& -\left(\frac{1}{2} \int_{0}^{1}\left(\widetilde{a}_{\alpha}^{U} \widetilde{c}_{f \alpha}^{L}+\widetilde{a}_{\alpha}^{L} \widetilde{c}_{f \alpha}^{U}\right) d \alpha-E\left[\widetilde{a} \widetilde{c}_{m}\right]\right. \\
& \left.+\frac{1}{2} \int_{0}^{1}\left(\widetilde{a}_{\alpha}^{U} \widetilde{c}_{r \alpha}^{L}+\widetilde{a}_{\alpha}^{L} \widetilde{c}_{r \alpha}^{U}\right) d \alpha\right) E_{3}+E\left[\widetilde{c}_{m}\right] \frac{B_{3}}{A}-\frac{B_{1}}{2 A} \\
& \times \int_{0}^{1}\left(\widetilde{\beta}_{\alpha}^{U} \widetilde{c}_{m \alpha}^{L}+\widetilde{\beta}_{\alpha}^{L} \widetilde{c}_{m \alpha}^{U}\right) d \alpha+\left(E\left[\widetilde{c}_{f}\right]-E\left[\widetilde{c}_{m}\right]+E\left[\widetilde{c}_{r}\right]\right) \\
& \times\left(\frac{2 B_{3} E_{3}+2 B_{1} E_{1}}{A} w_{m}^{*}+\frac{B_{4} E_{3}+B_{3} E_{4}+B_{1} E_{2}+B_{2} E_{1}}{A}\right) \\
& -\left(\frac{1}{2} \int_{0}^{1}\left(\widetilde{\beta}_{\alpha}^{U} \widetilde{c}_{f \alpha}^{L}+\widetilde{\beta}_{\alpha}^{L} \widetilde{c}_{f \alpha}^{U}\right) d \alpha\right. \\
& \left.-E\left[\widetilde{\beta} \widetilde{c}_{m}\right]+\frac{1}{2} \int_{0}^{1}\left(\widetilde{\beta}_{\alpha}^{U} \widetilde{c}_{r \alpha}^{L}+\widetilde{\beta}_{\alpha}^{L} \widetilde{c}_{r \alpha}^{U}\right) d \alpha\right) \\
& \times\left(\frac{2 B_{1} E_{3}+2 B_{3} E_{1}}{A} w_{m}^{*}\right. \\
& \left.+\frac{B_{1} E_{4}+B_{2} E_{3}+B_{3} E_{2}+B_{4} E_{1}}{A}\right)=0
\end{aligned}
$$


where $A, B_{1}, B_{2}, B_{3}, B_{4}, E_{1}, E_{2}, E_{3}, E_{4}$ are defined as in Proposition 1, respectively.

Proof. With some manipulations, the expected value $E\left[\pi_{m}\right]$ of $\pi_{m}$, defined in (5), can be rewritten as follows:

$$
\begin{aligned}
& E\left[\pi_{m}\right]=\left(2 E[\tilde{a}]+(E[\tilde{\beta}]-1)\left(p_{1}+p_{2}\right)\right) w \\
& -\left(\frac{1}{2} \int_{0}^{1}\left(\widetilde{a}_{\alpha}^{U} \widetilde{c}_{f \alpha}^{L}+\widetilde{a}_{\alpha}^{L} \widetilde{c}_{f \alpha}^{U}\right) d \alpha-E\left[\widetilde{a} \widetilde{c}_{m}\right]\right. \\
& \left.+\frac{1}{2} \int_{0}^{1}\left(\widetilde{a}_{\alpha}^{U} \widetilde{c}_{r \alpha}^{L}+\tilde{a}_{\alpha}^{L} \widetilde{c}_{r \alpha}^{U}\right) d \alpha\right) \tau_{1} \\
& +\left(E\left[\widetilde{c}_{f}\right]-E\left[\widetilde{c}_{m}\right]+E\left[\widetilde{c}_{r}\right]\right) p_{1} \tau_{1} \\
& -\left(\frac{1}{2} \int_{0}^{1}\left(\widetilde{\beta}_{\alpha}^{U} \widetilde{c}_{f \alpha}^{L}+\widetilde{\beta}_{\alpha}^{L} \widetilde{c}_{f \alpha}^{U}\right) d \alpha-E\left[\widetilde{\beta} \widetilde{c}_{m}\right]\right. \\
& \left.+\frac{1}{2} \int_{0}^{1}\left(\widetilde{\beta}_{\alpha}^{U} \widetilde{c}_{r \alpha}^{L}+\widetilde{\beta}_{\alpha}^{L} \widetilde{c}_{r \alpha}^{U}\right) d \alpha\right) p_{2} \tau_{1} \\
& -\frac{1}{2} \int_{0}^{1}\left(\widetilde{a}_{\alpha}^{U} \widetilde{c}_{m \alpha}^{L}+\widetilde{a}_{\alpha}^{L} \widetilde{c}_{m \alpha}^{U}\right) d \alpha+E\left[\widetilde{c}_{m}\right] p_{1} \\
& -\frac{p_{2}}{2} \int_{0}^{1}\left(\widetilde{\beta}_{\alpha}^{U} \widetilde{c}_{m \alpha}^{L}+\widetilde{\beta}_{\alpha}^{L} \widetilde{c}_{m \alpha}^{U}\right) d \alpha \\
& -\left(\frac{1}{2} \int_{0}^{1}\left(\widetilde{a}_{\alpha}^{U} \widetilde{c}_{f \alpha}^{L}+\tilde{a}_{\alpha}^{L} \widetilde{c}_{f \alpha}^{U}\right) d \alpha-E\left[\widetilde{a} \widetilde{c}_{m}\right]\right. \\
& \left.+\frac{1}{2} \int_{0}^{1}\left(\widetilde{a}_{\alpha}^{U} \widetilde{c}_{r \alpha}^{L}+\widetilde{a}_{\alpha}^{L} \widetilde{c}_{r \alpha}^{U}\right) d \alpha\right) \tau_{2} \\
& +\left(E\left[\widetilde{c}_{f}\right]-E\left[\widetilde{c}_{m}\right]+E\left[\widetilde{c}_{r}\right]\right) p_{2} \tau_{2} \\
& -\left(\frac{1}{2} \int_{0}^{1}\left(\widetilde{\beta}_{\alpha}^{U} \widetilde{c}_{f \alpha}^{L}+\widetilde{\beta}_{\alpha}^{L} \widetilde{c}_{f \alpha}^{U}\right) d \alpha-E\left[\widetilde{\beta} \widetilde{c}_{m}\right]\right. \\
& \left.+\frac{1}{2} \int_{0}^{1}\left(\widetilde{\beta}_{\alpha}^{U} \widetilde{c}_{r \alpha}^{L}+\widetilde{\beta}_{\alpha}^{L} \widetilde{c}_{r \alpha}^{U}\right) d \alpha\right) p_{1} \tau_{2} \\
& -\frac{1}{2} \int_{0}^{1}\left(\widetilde{a}_{\alpha}^{U} \widetilde{c}_{m \alpha}^{L}+\widetilde{a}_{\alpha}^{L} \widetilde{c}_{m \alpha}^{U}\right) d \alpha+E\left[\widetilde{c}_{m}\right] p_{2} \\
& -\frac{p_{1}}{2} \int_{0}^{1}\left(\widetilde{\beta}_{\alpha}^{U} \widetilde{c}_{m \alpha}^{L}+\widetilde{\beta}_{\alpha}^{L} \widetilde{c}_{m \alpha}^{U}\right) d \alpha .
\end{aligned}
$$

With (6)-(9) and (16), the first order derivative of $E\left[\pi_{m}\right]$ to $w$ can be shown as

$$
\begin{aligned}
& \frac{\partial E\left[\pi_{m}\right]}{\partial w} \\
& =2 E[\widetilde{a}]+(E[\widetilde{\beta}]-1)\left(p_{1}^{*}+p_{2}^{*}\right)+w(E[\widetilde{\beta}]-1) \\
& \times\left(\frac{\partial p_{1}^{*}}{\partial w}+\frac{\partial p_{2}^{*}}{\partial w}\right)-\left(\frac{1}{2} \int_{0}^{1}\left(\widetilde{a}_{\alpha}^{U} \widetilde{c}_{f \alpha}^{L}+\widetilde{a}_{\alpha}^{L} \widetilde{c}_{f \alpha}^{U}\right) d \alpha-E\left[\widetilde{a}_{m}\right]\right. \\
& \left.+\frac{1}{2} \int_{0}^{1}\left(\widetilde{a}_{\alpha}^{U} \widetilde{c}_{r \alpha}^{L}+\widetilde{a}_{\alpha}^{L} \widetilde{c}_{r \alpha}^{U}\right) d \alpha\right) \frac{\partial \tau_{1}^{*}}{\partial w}
\end{aligned}
$$

$$
\begin{aligned}
& +\left(E\left[\widetilde{c}_{f}\right]-E\left[\widetilde{c}_{m}\right]+E\left[\widetilde{c}_{r}\right]\right)\left(\tau_{1}^{*} \frac{\partial p_{1}^{*}}{\partial w}+p_{1}^{*} \frac{\partial \tau_{1}^{*}}{\partial w}\right) \\
& -\left(\frac{1}{2} \int_{0}^{1}\left(\widetilde{\beta}_{\alpha}^{U} \widetilde{c}_{f \alpha}^{L}+\widetilde{\beta}_{\alpha}^{L} \widetilde{c}_{f \alpha}^{U}\right) d \alpha-E\left[\widetilde{\beta} \widetilde{c}_{m}\right]\right. \\
& \left.+\frac{1}{2} \int_{0}^{1}\left(\widetilde{\beta}_{\alpha}^{U} \widetilde{c}_{r \alpha}^{L}+\widetilde{\beta}_{\alpha}^{L} \widetilde{c}_{r \alpha}^{U}\right) d \alpha\right) \\
& \times\left(\tau_{1}^{*} \frac{\partial p_{2}^{*}}{\partial w}+p_{2}^{*} \frac{\partial \tau_{1}^{*}}{\partial w}\right)+E\left[\widetilde{c}_{m}\right] \frac{\partial p_{1}^{*}}{\partial w}-\frac{\partial p_{2}^{*}}{\partial w} \\
& \times \frac{1}{2} \int_{0}^{1}\left(\widetilde{\beta}_{\alpha}^{U} \widetilde{c}_{m \alpha}^{L}+\widetilde{\beta}_{\alpha}^{L} \widetilde{c}_{m \alpha}^{U}\right) d \alpha \\
& -\left(\frac{1}{2} \int_{0}^{1}\left(\widetilde{a}_{\alpha}^{U} \widetilde{c}_{f \alpha}^{L}+\widetilde{a}_{\alpha}^{L} \widetilde{c}_{f \alpha}^{U}\right) d \alpha-E\left[\widetilde{a} \widetilde{c}_{m}\right]\right. \\
& \left.+\frac{1}{2} \int_{0}^{1}\left(\widetilde{a}_{\alpha}^{U} \widetilde{c}_{r \alpha}^{L}+\widetilde{a}_{\alpha}^{L} \widetilde{c}_{r \alpha}^{U}\right) d \alpha\right) \frac{\partial \tau_{2}^{*}}{\partial w} \\
& +\left(E\left[\widetilde{c}_{f}\right]-E\left[\widetilde{c}_{m}\right]+E\left[\widetilde{c}_{r}\right]\right)\left(\tau_{2}^{*} \frac{\partial p_{2}^{*}}{\partial w}+p_{2}^{*} \frac{\partial \tau_{2}^{*}}{\partial w}\right) \\
& -\left(\frac{1}{2} \int_{0}^{1}\left(\widetilde{\beta}_{\alpha}^{U} \widetilde{c}_{f \alpha}^{L}+\widetilde{\beta}_{\alpha}^{L} \widetilde{c}_{f \alpha}^{U}\right) d \alpha-E\left[\widetilde{\beta} \widetilde{c}_{m}\right]\right. \\
& \left.+\frac{1}{2} \int_{0}^{1}\left(\widetilde{\beta}_{\alpha}^{U} \widetilde{c}_{r \alpha}^{L}+\widetilde{\beta}_{\alpha}^{L} \widetilde{c}_{r \alpha}^{U}\right) d \alpha\right)\left(\tau_{2}^{*} \frac{\partial p_{1}^{*}}{\partial w}+p_{1}^{*} \frac{\partial \tau_{2}^{*}}{\partial w}\right) \\
& +E\left[\widetilde{c}_{m}\right] \frac{\partial p_{2}^{*}}{\partial w}-\frac{\partial p_{1}^{*}}{\partial w} \frac{1}{2} \int_{0}^{1}\left(\widetilde{\beta}_{\alpha}^{U} \widetilde{c}_{m \alpha}^{L}+\widetilde{\beta}_{\alpha}^{L} \widetilde{c}_{m \alpha}^{U}\right) d \alpha .
\end{aligned}
$$

Therefore, by setting (17) to zero, we can easily have (15).

Proposition 3. In the Manufacturer Stackelberg game case, the two competitive retailers' optimal retail prices (denoted as $p_{m 1}^{*}$ and $p_{m 2}^{*}$, resp.) and the optimal collecting rates (denoted as $\tau_{m 1}^{*}$ and $\tau_{m 2}^{*}$, resp.) are

$$
\begin{aligned}
p_{m 1}^{*} & =\frac{B_{1}}{A} w_{m}^{*}+\frac{B_{2}}{A}, \\
p_{m 2}^{*} & =\frac{B_{3}}{A} w_{m}^{*}+\frac{B_{4}}{A}, \\
\tau_{m 1}^{*} & =E_{1} w_{m}^{*}+E_{2}, \\
\tau_{m 2}^{*} & =E_{3} w_{m}^{*}+E_{4},
\end{aligned}
$$

where $A, B_{1}, B_{2}, B_{3}, B_{4}, E_{1}, E_{2}, E_{3}, E_{4}$ are defined as in Proposition 1, respectively. $w_{m}^{*}$ is defined as in (15).

Proof. By Propositions 1 and 2, we can easily see that Proposition 3 holds.

3.2. Retailer Stackelberg. The Retailer Stackelberg scenario arises in markets where the two competitive retailers' sizes are larger compared to their manufacturer. Because of their sizes, the two competitive retailers can maintain their margin on sales while squeezing profit from their suppliers. Similar 
game-theoretic framework as applied in the Manufacturer Stackelberg case is implemented to solve this problem. First, the manufacturer's problem is solved to derive the decision conditional on the retail prices and collecting rates chosen by the two competitive retailers. The two competitive retailers' problems are then solved given that the two competitive retailers know how the manufacturer would react to their retail prices and collecting rates.

Without loss of generality, let $m_{i}$ be the margin of retailer $i$ enjoyed by selling the new product, namely,

$$
p_{i}=w+m_{i}, \quad i=1,2,
$$

where $m_{i}>0$.

3.2.1. Manufacturer's Decision. Since the two competitive retailers move first in this game, we need to calculate the manufacturer's decision. The manufacturer is trying to maximize his own expected profit $E\left[\pi_{m}\right]$, where $\pi_{m}$ is defined as in (16).

Proposition 4. In the Retailer Stackelberg game case, the manufacturer's optimal decision, given retail prices $p_{1}$ and $p_{2}$ and the collecting rates $\tau_{1}$ and $\tau_{2}$, is

$$
w^{*}=F_{1}-\frac{1}{2} p_{1}-\frac{1}{2} p_{2}+F_{2}\left(\tau_{1}+\tau_{2}\right)
$$

where

$$
\begin{aligned}
F_{1}= & \frac{E[\widetilde{a}]+E\left[\widetilde{c}_{m}\right]-(1 / 2) \int_{0}^{1}\left(\widetilde{\beta}_{\alpha}^{U} \widetilde{c}_{m \alpha}^{L}+\widetilde{\beta}_{\alpha}^{L} \widetilde{c}_{m \alpha}^{U}\right) d \alpha}{1-E[\widetilde{\beta}]}, \\
F_{2}= & \left(E\left[\widetilde{c}_{f}\right]-E\left[\widetilde{c}_{m}\right]+E\left[\widetilde{c}_{r}\right]-\frac{1}{2} \int_{0}^{1}\left(\widetilde{\beta}_{\alpha}^{U} \widetilde{c}_{f \alpha}^{L}+\widetilde{\beta}_{\alpha}^{L} \widetilde{c}_{f \alpha}^{U}\right) d \alpha\right. \\
& \left.-\frac{1}{2} \int_{0}^{1}\left(\widetilde{\beta}_{\alpha}^{U} \widetilde{c}_{r \alpha}^{L}+\widetilde{\beta}_{\alpha}^{L} \widetilde{c}_{r \alpha}^{U}\right) d \alpha+E\left[\widetilde{\beta} \widetilde{c}_{m}\right]\right) \\
& \times(2(1-E[\widetilde{\beta}]))^{-1} .
\end{aligned}
$$

Proof. Using (16), we have the first order derivative of $E\left[\pi_{m}\right]$ to $w$ as follows:

$$
\begin{aligned}
& \frac{\partial E\left[\pi_{m}\right]}{\partial w} \\
& =2 E[\widetilde{a}]+(E[\widetilde{\beta}]-1)\left(p_{1}+p_{2}\right)+2(E[\tilde{\beta}]-1) w \\
& \quad+\left(E\left[\widetilde{c}_{f}\right]-E\left[\widetilde{c}_{m}\right]+E\left[\widetilde{c}_{r}\right]\right)\left(\tau_{1}+\tau_{2}\right) \\
& \quad-\left(\frac{1}{2} \int_{0}^{1}\left(\widetilde{\beta}_{\alpha}^{U} \widetilde{c}_{f \alpha}^{L}+\widetilde{\beta}_{\alpha}^{L} \widetilde{c}_{f \alpha}^{U}\right) d \alpha-E\left[\widetilde{\beta} \widetilde{c}_{m}\right]+2 E\left[\widetilde{c}_{m}\right]\right. \\
& \left.\quad+\frac{1}{2} \int_{0}^{1}\left(\widetilde{\beta}_{\alpha}^{U} \widetilde{c}_{r \alpha}^{L}+\widetilde{\beta}_{\alpha}^{L} \widetilde{c}_{r \alpha}^{U}\right) d \alpha\right)\left(\tau_{1}+\tau_{2}\right)
\end{aligned}
$$

$$
\begin{aligned}
& -\frac{1}{2} \int_{0}^{1}\left(\widetilde{a}_{\alpha}^{U} \widetilde{c}_{m \alpha}^{L}+\widetilde{a}_{\alpha}^{L} \widetilde{c}_{m \alpha}^{U}\right) d \alpha \\
& +E\left[\widetilde{c}_{m}\right] p_{2}-\frac{1}{2} \int_{0}^{1}\left(\widetilde{\beta}_{\alpha}^{U} \widetilde{c}_{m \alpha}^{L}+\widetilde{\beta}_{\alpha}^{L} \widetilde{c}_{m \alpha}^{U}\right) d \alpha .
\end{aligned}
$$

We can easily see that Proposition 4 holds, by setting (22) to zero and solving it.

3.2.2. Retailers' Decisions. Having the information about the decision of the manufacturer, each retailer would then use it to maximize her own expected profit $E\left[\pi_{r_{i}}\right]$, where $\pi_{r_{i}}$ is defined as in (11).

Note that the two competitive retailers move simultaneously. Therefore, we need to calculate the Nash decisions between them first.

Proposition 5. In the Retailer Stackelberg game case, the optimal retail price and collecting rate (denoted as $p_{r 1}^{*}$ and $\tau_{r 1}^{*}$, resp.) of retailer 1 and the optimal retail price and collecting rate (denoted as $p_{r 2}^{*}$ and $\tau_{r 2}^{*}$, resp.) of retailer 2 are given as follows:

$$
\begin{aligned}
p_{r 1}^{*}= & \frac{G_{1} G_{5}-G_{3} G_{4}}{G_{3} G_{6}-G_{2} G_{5}}, \\
p_{r 2}^{*}= & \frac{G_{1} G_{6}-G_{2} G_{4}}{G_{2} G_{5}-G_{3} G_{6}}, \\
\tau_{r 1}^{*}= & \frac{E\left[\widetilde{c}_{f} \tilde{a}\right]-F_{2} E[\widetilde{a}]}{2 E\left[\widetilde{k}_{1}\right]}+\frac{E\left[\widetilde{c}_{f} \widetilde{\beta}\right]-F_{2} E[\tilde{\beta}]}{2 E\left[\widetilde{k}_{1}\right]} p_{r 2}^{*} \\
& +\frac{F_{2}-E\left[\widetilde{c}_{f}\right]}{2 E\left[\widetilde{k}_{1}\right]} p_{r 1}^{*}, \\
\tau_{r 2}^{*}= & \frac{E\left[\widetilde{c}_{f} \tilde{a}\right]-F_{2} E[\widetilde{a}]}{2 E\left[\widetilde{k}_{2}\right]}+\frac{E\left[\widetilde{c}_{f} \tilde{\beta}\right]-F_{2} E[\tilde{\beta}]}{2 E\left[\widetilde{k}_{2}\right]} p_{r 1}^{*} \\
& +\frac{F_{2}-E\left[\widetilde{c}_{f}\right]}{2 E\left[\widetilde{k}_{2}\right]} p_{r 2}^{*},
\end{aligned}
$$

where

$$
\begin{aligned}
F_{1}= & \frac{E[\widetilde{a}]+E\left[\widetilde{c}_{m}\right]-(1 / 2) \int_{0}^{1}\left(\widetilde{\beta}_{\alpha}^{U} \widetilde{c}_{m \alpha}^{L}+\widetilde{\beta}_{\alpha}^{L} \widetilde{c}_{m \alpha}^{U}\right) d \alpha}{1-E[\tilde{\beta}]}, \\
F_{2}= & \left(E\left[\widetilde{c}_{f}\right]-E\left[\widetilde{c}_{m}\right]+E\left[\widetilde{c}_{r}\right]-\frac{1}{2} \int_{0}^{1}\left(\widetilde{\beta}_{\alpha}^{U} \widetilde{c}_{f \alpha}^{L}+\widetilde{\beta}_{\alpha}^{L} \widetilde{c}_{f \alpha}^{U}\right) d \alpha\right. \\
& \left.-\frac{1}{2} \int_{0}^{1}\left(\widetilde{\beta}_{\alpha}^{U} \widetilde{c}_{r \alpha}^{L}+\widetilde{\beta}_{\alpha}^{L} \widetilde{c}_{r \alpha}^{U}\right) d \alpha+E\left[\widetilde{\beta}_{m}\right]\right) \\
& \times(2(1-E[\tilde{\beta}]))^{-1},
\end{aligned}
$$




$$
\begin{aligned}
G_{1}= & F_{1}+\frac{3}{2} E[\widetilde{a}]+\frac{F_{2}\left(E\left[\widetilde{c}_{f} \tilde{a}\right]-F_{2} E[\tilde{a}]\right)}{2 E\left[\widetilde{k}_{2}\right]} \\
& +\frac{\left(F_{2}-E\left[\widetilde{c}_{f}\right]\right)\left(E\left[\widetilde{c}_{f} \tilde{a}\right]-F_{2} E[\widetilde{a}]\right)}{2 E\left[\widetilde{k}_{1}\right]} \\
G_{2}= & \frac{F_{2}\left(E\left[\widetilde{c}_{f} \widetilde{\beta}\right]-F_{2} E[\widetilde{\beta}]\right)}{2 E\left[\widetilde{k}_{2}\right]} \\
& +\frac{\left(F_{2}-E\left[\widetilde{c}_{f}\right]\right)^{2}}{2 E\left[\widetilde{k}_{1}\right]}-3, \\
G_{3}= & \frac{3 E[\tilde{\beta}]-1}{2} \\
& +\frac{\left(F_{2}-E\left[\widetilde{c}_{f}\right]\right)\left(E\left[\widetilde{c}_{f} \tilde{\beta}\right]-F_{2} E[\widetilde{\beta}]\right)}{2 E\left[\widetilde{k}_{1}\right]} \\
& +\frac{F_{2}\left(F_{2}-E\left[\widetilde{c}_{f}\right]\right)}{2 E\left[\widetilde{k}_{2}\right]} \\
G_{4}= & F_{1}+\frac{3 E[\widetilde{a}]}{2} \\
& +\frac{\left(F_{2}-E\left[\widetilde{c}_{f}\right]\right)\left(E\left[\widetilde{c}_{f} \tilde{a}\right]-F_{2} E[\widetilde{a}]\right)}{2 E\left[\widetilde{k}_{2}\right]} \\
2 E\left[\widetilde{k}_{1}\right] &
\end{aligned}
$$$$
G_{5}=\frac{F_{2}\left(E\left[\widetilde{c}_{f} \tilde{\beta}\right]-F_{2} E[\tilde{\beta}]\right)}{2 E\left[\widetilde{k}_{1}\right]}
$$$$
+\frac{\left(F_{2}-E\left[\widetilde{c}_{f}\right]\right)^{2}}{2 E\left[\widetilde{k}_{2}\right]}-3,
$$$$
G_{6}=\frac{3 E[\widetilde{\beta}]-1}{2}
$$$$
+\frac{\left(F_{2}-E\left[\widetilde{c}_{f}\right]\right)\left(E\left[\widetilde{c}_{f} \tilde{\beta}\right]-F_{2} E[\tilde{\beta}]\right)}{2 E\left[\widetilde{k}_{2}\right]}
$$$$
+\frac{F_{2}\left(F_{2}-E\left[\widetilde{c}_{f}\right]\right)}{2 E\left[\widetilde{k}_{1}\right]} .
$$

Solving (31), simultaneously, we can easily see that Proposition 5 holds.

Proposition 6. In the Retailer Stackelberg game case, the manufacturer's optimal decision (denoted as $w_{r}^{*}$ ) is

$$
w_{r}^{*}=F_{1}-\frac{1}{2} p_{r 1}^{*}-\frac{1}{2} p_{r 2}^{*}+F_{2}\left(\tau_{r 1}^{*}+\tau_{r 2}^{*}\right),
$$

where $p_{r 1}^{*}, p_{r 2}^{*}, \tau_{r 1}^{*}, \tau_{r 2}^{*}$ are defined as in (23)-(26), respectively, and

$$
\begin{aligned}
F_{1}= & \frac{E[\widetilde{a}]+E\left[\widetilde{c}_{m}\right]-(1 / 2) \int_{0}^{1}\left(\widetilde{\beta}_{\alpha}^{U} \widetilde{c}_{m \alpha}^{L}+\widetilde{\beta}_{\alpha}^{L} \widetilde{c}_{m \alpha}^{U}\right) d \alpha}{1-E[\widetilde{\beta}]} \\
F_{2}= & \left(E\left[\widetilde{c}_{f}\right]-E\left[\widetilde{c}_{m}\right]+E\left[\widetilde{c}_{r}\right]-\frac{1}{2} \int_{0}^{1}\left(\widetilde{\beta}_{\alpha}^{U} \widetilde{c}_{f \alpha}^{L}+\widetilde{\beta}_{\alpha}^{L} \widetilde{c}_{f \alpha}^{U}\right) d \alpha\right. \\
& \left.-\frac{1}{2} \int_{0}^{1}\left(\widetilde{\beta}_{\alpha}^{U} \widetilde{c}_{r \alpha}^{L}+\widetilde{\beta}_{\alpha}^{L} \widetilde{c}_{r \alpha}^{U}\right) d \alpha+E\left[\widetilde{\beta} \widetilde{c}_{m}\right]\right) \\
& \times(2(1-E[\widetilde{\beta}]))^{-1} .
\end{aligned}
$$

Proof. By Propositions 4 and 5, we can easily see that Proposition 6 holds. 
TABLE 1: Relation between linguistic expression and triangular fuzzy variable.

\begin{tabular}{|c|c|c|}
\hline & Linguistic expression & Triangular fuzzy variable \\
\hline \multirow{3}{*}{ Remanufacturing cost $\widetilde{c}_{r}$} & Low (about 7) & $(6,7,9)$ \\
\hline & Medium (about 11) & $(9,11,14)$ \\
\hline & High (about 16) & $(14,16,19)$ \\
\hline \multirow{3}{*}{ Manufacturing $\operatorname{cost} \widetilde{c}_{m}$} & Low (about 17) & $(15,17,20)$ \\
\hline & Medium (about 23) & $(20,23,25)$ \\
\hline & High (about 29) & $(25,29,35)$ \\
\hline \multirow{2}{*}{ Market base $\tilde{a}$} & Large (about 400) & $(300,400,450)$ \\
\hline & Small (about 200) & $(150,200,280)$ \\
\hline \multirow{2}{*}{ Price elasticity $\widetilde{\beta}$} & Very sensitive (about 0.8 ) & $(0.6,0.8,0.9)$ \\
\hline & Sensitive (about 0.5) & $(0.3,0.5,0.6)$ \\
\hline \multirow{3}{*}{ Taking back transfer cost $\widetilde{c}_{f}$} & Low (about 2) & $(1,2,3)$ \\
\hline & Medium (about 4) & $(3,4,5)$ \\
\hline & High (about 6) & $(5,6,8)$ \\
\hline \multirow{3}{*}{ Scaling parameter $\widetilde{k}_{1}$} & Low (about 500) & $(450,500,650)$ \\
\hline & Medium (about 800) & $(700,800,1000)$ \\
\hline & High (about 1100) & $(1000,1100,1300)$ \\
\hline \multirow{3}{*}{ Scaling parameter $\widetilde{k}_{2}$} & Low (about 550) & $(400,550,650)$ \\
\hline & Medium (about 850) & $(650,850,1000)$ \\
\hline & High (about 1200) & $(1000,1200,1300)$ \\
\hline
\end{tabular}

3.3. Vertical Nash. In the Vertical Nash model, every firm has equal bargaining power and thus they make their decisions simultaneously. This scenario arises in a market in which there are relatively small- to medium-sized manufacturers and retailers. Since a manufacturer cannot dominate the market over the two competitive retailers, his price decision is conditioned on how the two competitive retailers price the new product. On the other hand, the two competitive retailers must also condition their own retail price and own collecting rate decisions on the wholesale price.

Consider that the decisions of the two competitive retailers and the manufacturer are already derived in the Manufacturer Stackelberg and Retailer Stackelberg game cases, respectively. From the Manufacturer Stackelberg game, the two competitive retailers' decisions for given wholesale price $w$ are given in (6)-(9). From the Retailer Stackelberg game, the manufacturer's decision for given retail prices $p_{1}$ and $p_{2}$ and the collecting rates $\tau_{1}$ and $\tau_{2}$ is given in (20).

Solving (6)-(9) and (20) simultaneously yields the Nash decision solution. The optimal Nash decisions can be derived and be given Proposition 7 .

Proposition 7. In the Vertical Nash case, the optimal retail prices (denoted as $p_{n 1}^{*}$ and $p_{n 2}^{*}$ ) chosen by retailer 1 and retailer 2 , respectively, the optimal collecting rates (denoted as $\tau_{n 1}^{*}$ and $\tau_{n 2}^{*}$ ) chosen by retailer 1 and retailer 2 , respectively, and the optimal wholesale price (denoted as $w_{n}^{*}$ ) chosen by the manufacturer are

$$
\begin{gathered}
w_{n}^{*}=\frac{A F_{1}+F_{2}\left(B_{2}+B_{4}\right)+A F_{3}\left(E_{2}+E_{4}\right)}{A-F_{2}\left(B_{1}+B_{3}\right)-A F_{2}\left(E_{1}+E_{3}\right)}, \\
p_{1}^{*}=\frac{B_{1}}{A} w_{n}^{*}+\frac{B_{2}}{A},
\end{gathered}
$$

TABLE 2: Optimal expected profits of the manufacturer and the two retailers.

\begin{tabular}{lccc}
\hline Game scenario & $E\left[\pi_{m}\right]$ & $E\left[\pi_{r_{1}}\right]$ & $E\left[\pi_{r_{2}}\right]$ \\
\hline Manufacturer Stackelberg & 95194 & 15161 & 15159 \\
Retailer Stackelberg & 76463 & 26996 & 27519 \\
Vertical Nash & 91005 & 21868 & 21865 \\
\hline
\end{tabular}

$$
\begin{aligned}
p_{2}^{*} & =\frac{B_{3}}{A} w_{n}^{*}+\frac{B_{4}}{A}, \\
\tau_{1}^{*} & =E_{1} w_{n}^{*}+E_{2}, \\
\tau_{2}^{*} & =E_{3} w_{n}^{*}+E_{4},
\end{aligned}
$$

where $A, B_{1}, B_{2}, B_{3}, B_{4}, E_{1}, E_{2}, E_{3}, E_{4}, F_{1}, F_{2}, F_{3}$ are defined as in Propositions 1 and 4, respectively.

Proof. Solving (6)-(9) and (20), simultaneously, we can see that Proposition 7 holds.

\section{Numerical Studies}

In this section, we compare the results obtained from the above three different decision scenarios using numerical approach and study the behavior of firms facing changing environment. By the results obtained from the above three different decision scenarios, we can easily see the expressions of the optimal wholesale price, retail prices, collected rates, and optimal expected profits under different decision scenarios. 
TABLE 3: Optimal decisions of retail prices, wholesale price, and collecting rates.

\begin{tabular}{|c|c|c|c|c|c|}
\hline Game scenario & $p_{1}^{*}$ & $p_{2}^{*}$ & $w^{*}$ & $\tau_{1}^{*}$ & $\tau_{2}^{*}$ \\
\hline Manufacturer Stackelberg & 503.0028 & 503.0108 & 380.8939 & 0.3296 & 0.3247 \\
\hline Retailer Stackelberg & 484.3593 & 481.2045 & 279.4503 & 0.6861 & 0.6988 \\
\hline Vertical Nash & 455.7510 & 455.7604 & 309.0691 & 0.3883 & 0.3825 \\
\hline
\end{tabular}

Here, assume that the relationship between linguistic expressions and triangular fuzzy variables for manufacturing cost, remanufacturing cost, market base, scaling parameter, collecting transfer cost, and price elasticity is determined by experts' experiences as shown in Table 1.

Consider the case that the remanufacturing and manufacturing costs $\widetilde{c}_{r}$ and $\widetilde{c}_{m}$ are high $\left(\widetilde{c}_{r}\right.$ is about $16, \widetilde{c}_{m}$ is about $29)$, the market base $\tilde{a}$ is large ( $\widetilde{a}$ is about 400$)$, price elasticity $\widetilde{\beta}$ is sensitive $(\widetilde{\beta}$ is about 0.5$)$, taking back transfer cost $\widetilde{c}_{f}$ is medium $\left(\widetilde{c}_{f}\right.$ is about 4$)$, and scaling parameters $\widetilde{k}_{1}$ and $\widetilde{k}_{2}$ are medium $\left(\tilde{k}_{1}\right.$ is about $800, \widetilde{k}_{2}$ is about 850$)$. Using Table 1 , $\widetilde{c}_{r}=(14,16,19), \widetilde{c}_{m}=(25,29,35), \widetilde{a}=(300,400,450), \widetilde{\beta}=$ $(0.3,0.5,0.6), \widetilde{c}_{f}=(3,4,5), \widetilde{k}_{1}=(700,800,1000), \widetilde{k}_{2}=$ $(650,850,1000)$. The expected values are $E\left[\widetilde{c}_{r}\right]=(14+2 \times 16+$ 19) $/ 4=65 / 4, E\left[\widetilde{c}_{m}\right]=(25+2 \times 29+35) / 4=118 / 4, E[\widetilde{a}]=$ $(300+2 \times 400+450) / 4=1550 / 4, E[\widetilde{\beta}]=(0.3+2 \times 0.5+0.6) / 4=$ $1.9 / 4, E\left[\widetilde{c}_{f}\right]=(3+2 \times 4+5) / 4=4, E\left[\widetilde{k}_{1}\right]=(700+2 \times 800+$ $1000) / 4=3300 / 4, E\left[\widetilde{k}_{2}\right]=(650+2 \times 850+1000) / 4=3350 / 4$. The $\alpha$-optimistic value and $\alpha$-pessimistic value of $\widetilde{c}_{r}, \widetilde{c}_{m}, \widetilde{c}_{f}$, $\widetilde{\beta}$ and $\tilde{a}$ are $\widetilde{c}_{r \alpha}^{L}=14+2 \alpha, \widetilde{c}_{r \alpha}^{U}=19-3 \alpha, \widetilde{c}_{m \alpha}^{L}=25+4 \alpha, \widetilde{c}_{m \alpha}^{U}=$ $35-6 \alpha, \widetilde{\beta}_{\alpha}^{L}=0.3+0.2 \alpha, \widetilde{\beta}_{\alpha}^{U}=0.6-0.1 \alpha, \widetilde{c}_{f \alpha}^{L}=3+\alpha, \widetilde{c}_{f \alpha}^{U}=5-\alpha$, $\tilde{a}_{\alpha}^{L}=300+100 \alpha, \tilde{a}_{\alpha}^{U}=450-50 \alpha$, respectively. The results of expected profits and optimal decisions are shown as in Tables 2 and 3.

\section{Observation}

From Tables 2 and 3, we have the following results.

(1) For the three decentralized decision cases, the firm who is the leader in the supply chain has the advantage to get the higher profit; for example, the manufacturer's profit under Manufacturer Stackelberg game scenario is higher than that under Retailer Stackelberg game scenario and the Vertical Nash game case. For the two competitive retailers, they have their own minimal expected profits under Manufacturer Stackelberg game scenario.

(2) The new product's optimal retail prices charged by the two competitive retailers, respectively, under Vertical Nash decision case are lower than those under the Manufacturer Stackelberg and Retailer Stackelberg decision cases, and the optimal retail prices achieve the biggest value under Manufacturer Stackelberg game scenario.
(3) The new product achieves the highest wholesale price in the Manufacturer Stackelberg game, followed by the Vertical Nash game and then the Retailer Stackelberg game case.

(4) The optimal collecting rates of the used products charged by the two competitive retailers, respectively, achieve the highest wholesale price in the Retailer Stackelberg game, followed by the Vertical Nash game and then the Manufacturer Stackelberg game case.

\section{Conclusions}

Different from the conventional studies, this paper explores the roles of the two competitive retailers and the manufacturer and their bargaining powers by examining the supply chain in a fuzzy environment over three different game scenarios. We derive the expressions for optimal retail prices, wholesale price, and collecting rates with expected value model. By analyzing a numerical example, we further analyze the analytical solutions and give some managerial analysis.

Compared to the traditional approach used in the study of closed-loop supply chain, the proposed approach in this paper requires less data to model the fuzziness which is associated with the consumer demand, the manufacturing and remanufacturing costs of new product, and the collecting cost of the used product and can make use of the subjective estimation based on decision maker's judgment, experience, and intuitions. It is appropriate when the situation is ambiguous and lacks historical data.

However, we have made some assumptions that may be relaxed to improve the model in the future research. One assumption is that the demand function is linear; further work is desirable to test whether our conclusions extend to other forms of demand function. The other assumptions are that the closed-loop supply chains only with one period and competition only existing in retail process. Thus, the supply chain with competitive manufacturers and/or competitive retailers, and the model over multiple periods can be considered in the future.

\section{Acknowledgments}

The authors wish to express their sincerest thanks to the editors and anonymous referees for their constructive comments and suggestions on this paper. This research was supported in part by National Natural Science Foundation of China, nos. 71001106, 70971069, and 71002106. 


\section{References}

[1] S. Dowlatshahi, "A strategic framework for the design and implementation of remanufacturing operations in reverse logistics," International Journal of Production Research, vol. 43, no. 16, pp. 3455-3480, 2005.

[2] S. Rubio and A. Corominas, "Optimal manufacturing-remanufacturing policies in a lean production environment," Computers and Industrial Engineering, vol. 55, no. 1, pp. 234-242, 2008.

[3] I. Konstantaras and S. Papachristos, "Optimal policy and holding cost stability regions in a periodic review inventory system with manufacturing and remanufacturing options," European Journal of Operational Research, vol. 178, no. 2, pp. 433-448, 2007.

[4] V. D. R. Guide, V. Jayaraman, and J. D. Linton, "Building contingency planning for closed-loop supply chains with product recovery," Journal of Operations Management, vol. 21, no. 3, pp. 259-279, 2003.

[5] M. E. Ketzenberg, E. Van Der Laan, and R. H. Teunter, "Value of information in closed loop supply chains," Production and Operations Management, vol. 15, no. 3, pp. 393-406, 2006.

[6] H. J. Zimmermann, "Application-oriented view of modeling uncertainty," European Journal of Operational Research, vol. 122, no. 2, pp. 190-198, 2000.

[7] L. A. Zadeh, "Fuzzy sets," Information and Computation, vol. 8, pp. 338-353, 1965.

[8] S. Nahmias, "Fuzzy variables," Fuzzy Sets and Systems, vol. 1, no. 2, pp. 97-110, 1978.

[9] A. Kaufmann and M. M. Gupta, Introduction to Fuzzy Arithmetic: Theory and Applications, Van Nostrand Reinhold, New York, NY, USA, 1985.

[10] B. Liu, "A survey of credibility theory," Fuzzy Optimization and Decision Making, vol. 5, no. 4, pp. 387-408, 2006.

[11] B. Liu and Y. K. Liu, "Expected value of fuzzy variable and fuzzy expected value models," IEEE Transactions on Fuzzy Systems, vol. 10, no. 4, pp. 445-450, 2002.

[12] J. Wang and Y.-F. Shu, "Fuzzy decision modeling for supply chain management," Fuzzy Sets and Systems. An International Journal in Information Science and Engineering, vol. 150, no. 1, pp. 107-127, 2005.

[13] J. Wei and J. Zhao, "Reverse channel decisions for a fuzzy closedloop supply chain," Applied Mathematical Modelling, vol. 37, pp. 1502-1513, 2013.

[14] D. Petrovic, "Simulation of supply chain behaviour and performance in an uncertain environment," International Journal of Production Economics, vol. 71, no. 1-3, pp. 429-438, 2001.

[15] S. Mondal and M. Maiti, "Multi-item fuzzy EOQ models using genetic algorithm," Computers and Industrial Engineering, vol. 44, no. 1, pp. 105-117, 2003.

[16] J. Zhao, W. Tang, and J. Wei, "Pricing decision for substitutable products with retail competition in a fuzzy environment," International Journal of Production Economics, vol. 135, no. 1, pp. 144-153, 2012.

[17] L. Li, S. N. Kabadi, and K. P. K. Nair, "Fuzzy models for singleperiod inventory problem," Fuzzy Sets and Systems, vol. 132, no. 3, pp. 273-289, 2002.

[18] S. K. Mukhopadhyay and H. Ma, "Joint procurement and production decisions in remanufacturing under quality and demand uncertainty," International Journal of Production Economics, vol. 120, no. 1, pp. 5-17, 2009.
[19] C. Kao and W.-K. Hsu, "A single-period inventory model with fuzzy demand," Computers \& Mathematics with Applications, vol. 43, no. 6-7, pp. 841-848, 2002.

[20] R. C. Savaskan, S. Bhattacharya, and L. N. Van Wassenhove, "Closed-loop supply chain models with product remanufacturing," Management Science, vol. 50, no. 2, pp. 239-252, 2004.

[21] C. Ingene and M. Parry, "Channel coordination when retailers compete," Marketing Science, vol. 14, no. 4, pp. 360-377, 1995.

[22] W. Hu and Y. Li, "Retail service for mixed retail and e-tail channels," Annals of Operations Research, vol. 192, pp. 151-171, 2012.

[23] R. Desiraju and S. Moorthy, "Managing a distribution channel under asymmetric information with performance requirements," Management Science, vol. 43, no. 12, pp. 1628-1644, 1997.

[24] S. C. Choi, "Price competition in a duopoly common retailer channel," Journal of Retailing, vol. 72, no. 2, pp. 117-134, 1996.

[25] A. Dixit, "A model of duopoly suggesting a theory of entry barriers," Bell Journal of Economics, vol. 10, pp. 20-32, 1979.

[26] N. Singh and X. Vives, "Price and quantity competition in a differentiated duopoly," Rand Journal of Economics, vol. 15, no. 4, pp. 546-554, 1984.

[27] X. Vives, "On the efficiency of Bertrand and Cournot equilibria with product differentiation," Journal of Economic Theory, vol. 36, no. 1, pp. 166-175, 1985. 


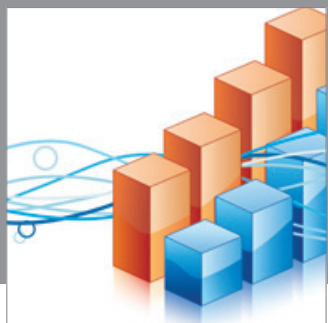

Advances in

Operations Research

mansans

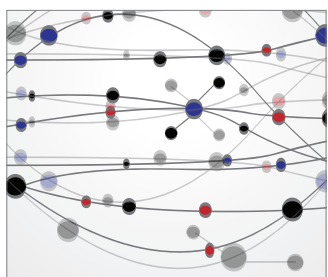

The Scientific World Journal
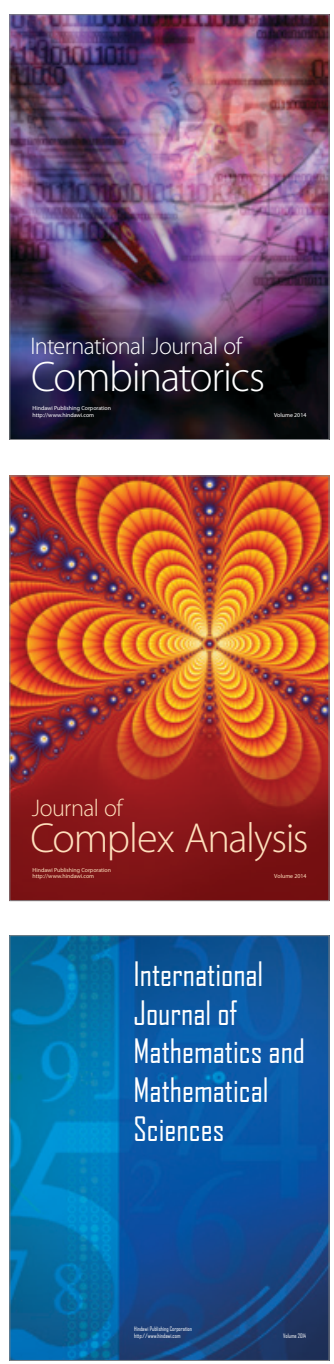
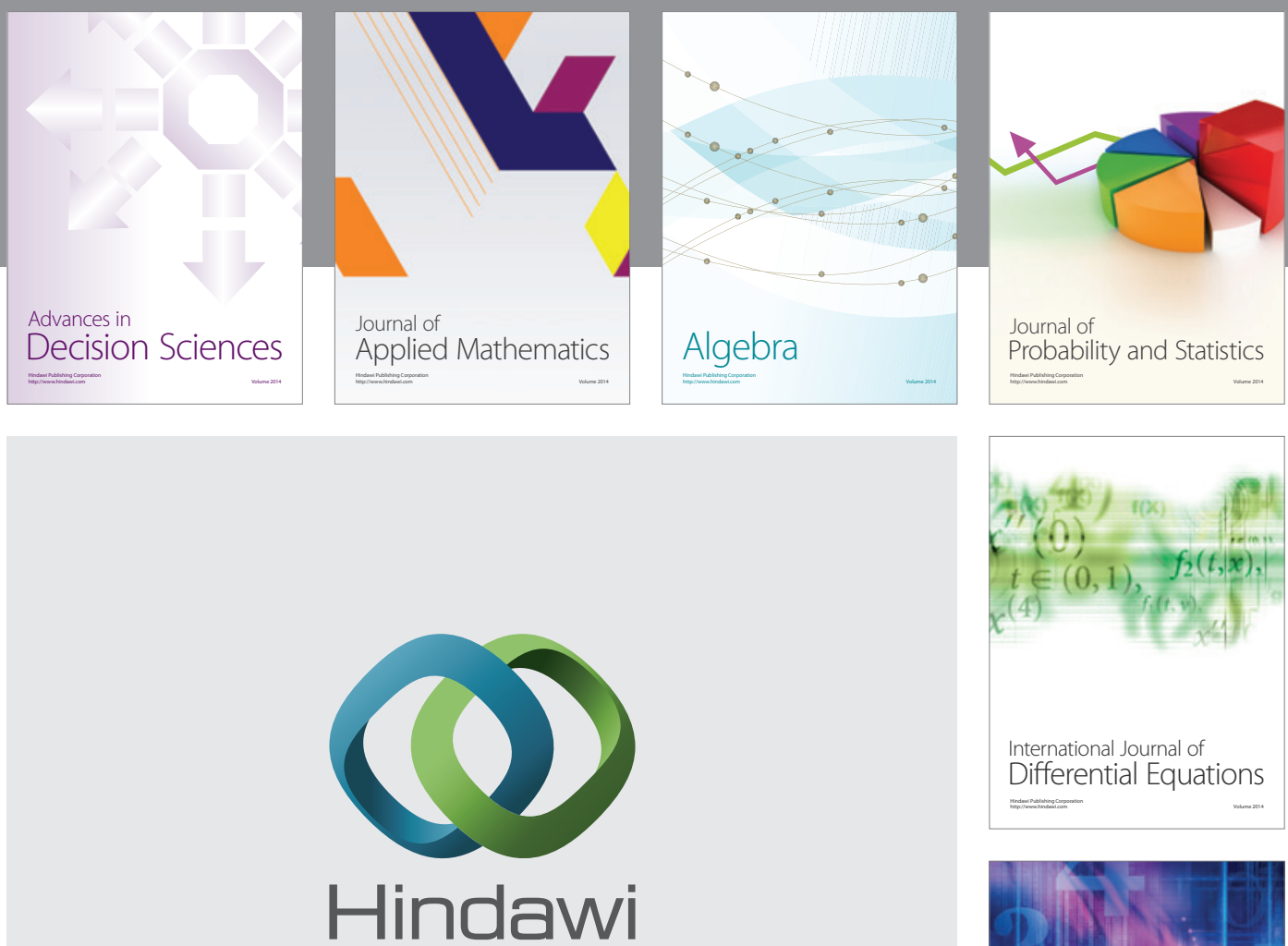

Submit your manuscripts at http://www.hindawi.com
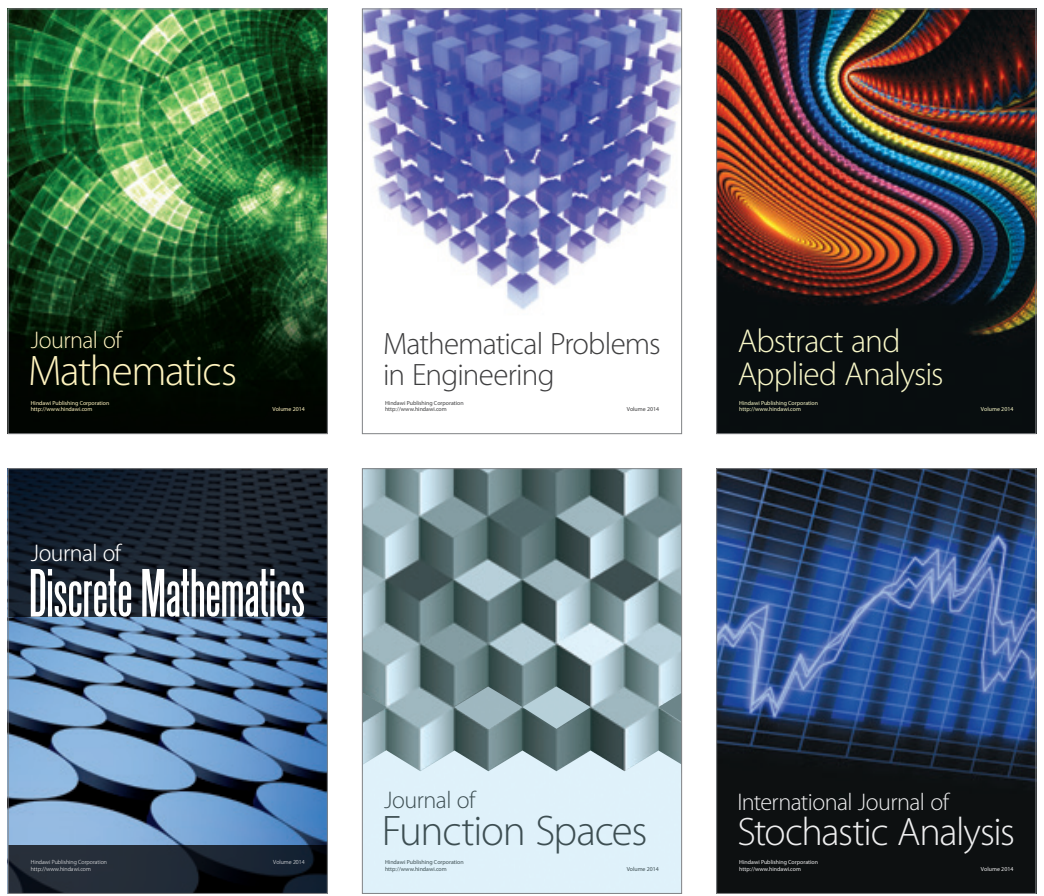

Journal of

Function Spaces

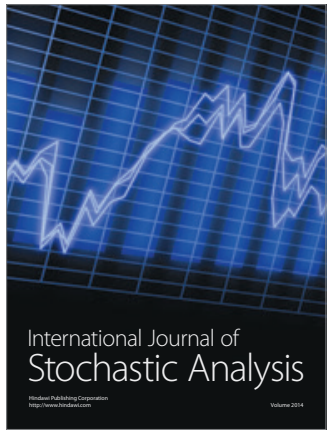

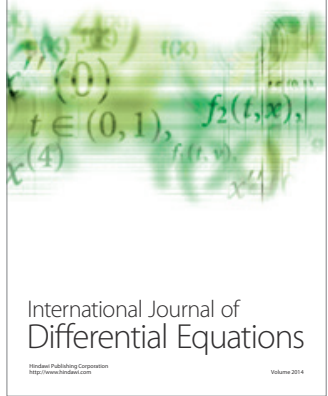
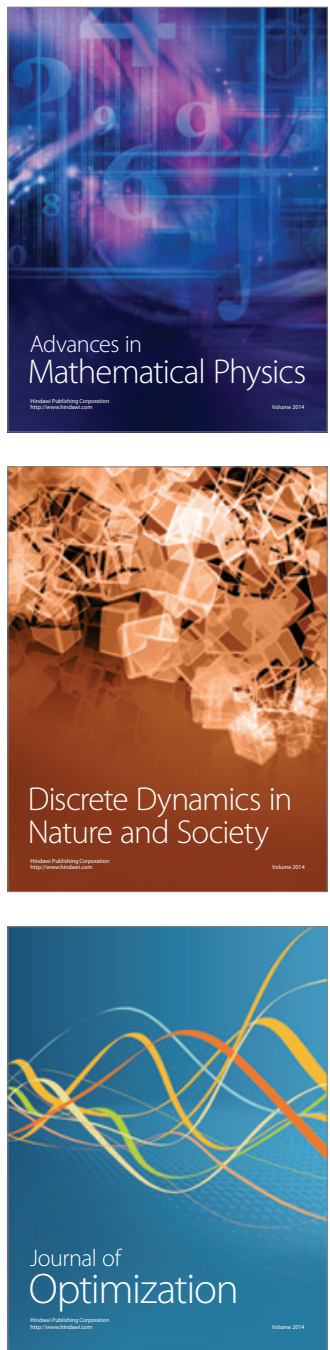